\title{
A design on intelligent public trash can based on machine vision and auxiliary sensors
}

\author{
Longyu Gao*, Leixin Han, Jiangyu Wu, Mingfei Liu, Ruming Kang \\ Tianjin University of Science and Technology, China; \\ E-mail:*ggafcc@163.com \\ www.tust.edu.cn
}

\begin{abstract}
In order to improve the correct rate of front-end recognition in the garbage classification process, the automatic garbage classification system designed based on machine vision technology has a significant improvement in recognition accuracy compared to traditional smart garbage cans. But in the case of identifying irregular garbage, the recognition accuracy is greatly reduced. In order to solve this kind of problem, four types of auxiliary sensors are added to the trash can, through the mutual cooperation between the sensors, combined with the results of machine vision recognition, comprehensive judgment, greatly improving the recognition accuracy of irregular garbage.
\end{abstract}

Keywords: machine vision, auxiliary sensors, garbage sorting

\section{Introduction}

With the rapid development of society, the level of consumption and production has greatly improved. At the same time, waste production and environmental degradation have also increased. According to statistics, the world produces approximately 10 billion tons of waste each year. 400 million tons, if garbage cannot be classified correctly, the land resources occupied by long-term stacking will exceed 25 million square meters, so the task of garbage classification is very difficult.

Garbage sorting can be divided into two parts: front-end sorting collection and back-end sorting and recycling. At present, the world's main front-end method of garbage classification still relies on manual work, and mainly relies on citizens' consciousness and staff review. This method is very inefficient, difficult to solve the problem of large amounts of untreated garbage, and cannot guarantee the correctness rate. Therefore, in the past two years, the concept of a smart trash can was proposed.

The current smart trash cans are mostly based on infrared sensors, capacitive sensors or LCD screens for identification and classification. Some smart trash cans have introduced machine vision and machine learning related technologies. However, due to the wide variety of garbage and the same type of style and Abundant shape changes make recognition difficult, and current methods cannot meet the needs of daily intelligent recognition and classification 1. Although some related studies can achieve a stable distinction between recyclable and non-recyclable garbage, they have not yet been able to meet China's need to distinguish four types of garbage.

In order to meet China's garbage classification requirements and improve the accuracy of front-end recognition in the garbage classification process, this design adds four types of sensors to the trash can, through the mutual cooperation between the sensors, combined with the results of machine vision recognition. Recognition and judgment have successfully achieved a substantial increase in the recognition accuracy of irregular garbage. 


\section{Overall design scheme of the system}

The intelligent waste classification system is mainly composed of machine vision module, auxiliary sensor module and mechanical module that realizes automatic classification control. When the garbage is put into the system, the machine vision module starts the camera to obtain the garbage image, and then runs the convolutional neural network to obtain the garbage category information. For garbage that cannot be identified, auxiliary sensors are used for identification. Finally, according to the classification of the garbage type, the mechanical structure is controlled to make the garbage fall into the corresponding bin ${ }^{2}$. In the auxiliary sensor part, we have selected 4 auxiliary sensors for the commonality of different types of garbage, namely VOC sensors, metal sensors, capacitive proximity sensors, and humidity sensors. The result of machine vision recognition is shown in Figure 1.

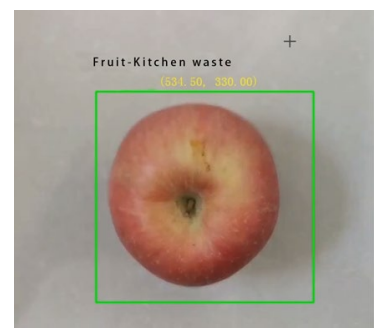

Fig.1. Machine vision recognition results

\subsection{VOC sensor}

The detection of hazardous waste that cannot be recognized by machine vision is mainly realized by the ZP16 digital VOC air quality sensor module. The air quality module uses advanced chip thick film semiconductor gas sensors. The gas sensors are resistant to formaldehyde, benzene, carbon monoxide, and ammonia., Hydrogen, alcohol, cigarette smoke, flavors and other organic volatile gases have extremely high sensitivity. After aging, debugging, calibration and calibration, the module has good consistency and extremely high sensitivity. Extremely high sensitivity, excellent long-term stability, factory calibration, convenient and quick to use, with sensor fault self-diagnosis function, low power consumption and long life. The VOC sensor is shown in Figure 2.

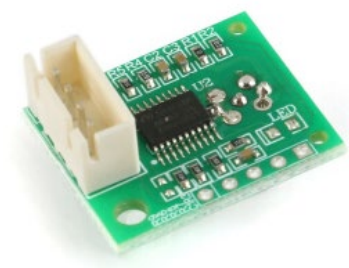

Fig.2. VOC sensor

\subsection{Metal sensor}

The metal sensor is used to identify metals and realize the function of material identification. This system uses LJ18A3-8-Z/BX type metal sensor. The sensor has the advantages of fast response speed, strong anti-interference ability, waterproof and corrosion resistance, and very stable performance. It is widely used in automated assembly lines, intelligent hardware and security systems. The operating voltage of the sensor is 6-36vdc, and the switch contains a high-frequency oscillation circuit, a detection circuit, an amplifying circuit, a de-transmitting circuit and an output circuit. When power is supplied to the switch, the oscillator in the high-frequency oscillation circuit generates an alternating electromagnetic field on the detection surface of the switch. When a metal approaches the detection surface of the switch, the eddy current inside the metal absorbs the energy of the alternating electromagnetic field in the oscillator, so that the oscillator weakens or stops. The two states of the energy change of the oscillator are converted into a level signal by the detection circuit, the level signal is amplified by the amplifying circuit, and then the trigger circuit triggers the output transistor circuit to work to generate a switching signal. Thereby detecting the presence or absence of metal to achieve the purpose of detecting metal. The metal sensor is shown in Figure 3.

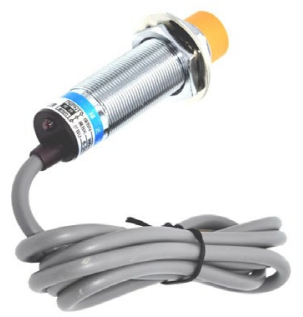

Fig.3. Metal sensor 


\subsection{Capacitive proximity sensor}

The second part of the material identification is mainly realized by the capacitive proximity switch M8M12. It can detect any dielectric substance, including conductors, semiconductors, insulators, and can even be used to detect liquid level and powdered materials. For non-metallic substances, the movement distance depends on the dielectric constant of the material. The greater the dielectric constant of the material, the farther the sensing distance is. Cooperating with motor control, changing the distance between it and the tray can realize the classification of different materials. The capacitive proximity sensor is shown in Figure 4.

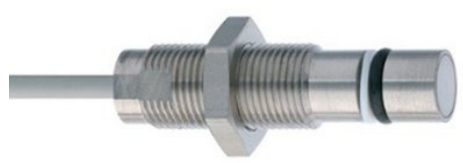

Fig.4. Capacitive proximity sensor

\subsection{Humidity Sensor}

Use SHT3x temperature and humidity sensor module to communicate with STM32, and turn it on when machine vision can't recognize it, so as to realize the identification of wet garbage. The module applies digital module collection technology and temperature and humidity sensing technology, and the product has high reliability and long-term stability. Built-in humidity and temperature sensor elements, analog to digital converters, signal processing, calibration data and I2C host interface. The technology of using low-k polymer dielectric for humidity sensing creates a low-power, monolithic MoS sensor module with low deviation and hysteresis and long-term stability. It is suitable for measuring humidity, dew point and temperature. The module is small in size and the accuracy can reach $\pm 0.4^{\circ} \mathrm{C}$. It can be embedded in many highly integrated environments. With millisecond measurement conversion time, there is no need to wait between starting the measurement and reading the data. The $2.54 \mathrm{~mm}$ spacing interface is convenient for integration testing. The humidity sensor is shown in Figure 5.

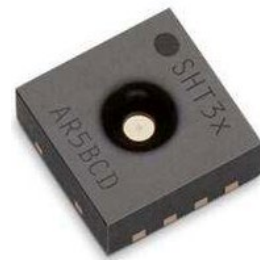

Fig.5. Humidity Sensor

\section{Auxiliary sensor recognition}

First, the VOC sensor detects the harmful gas produced by the corrupted hazardous garbage, and if it is detected, the garbage is marked as hazardous garbage. If no harmful gas is detected, the metal sensor is used to realize the function of material identification and distinguish between recyclable metals and others. If the metal is not recyclable, the capacitive proximity sensor is used to determine whether it is paper or plastic, and the paper and plastic are marked as recyclable. Finally, the humidity sensor is used to distinguish food waste from other wastes based on the characteristics of food waste containing more water, and mark them. The auxiliary sensor recognition process is shown in Figure 6.

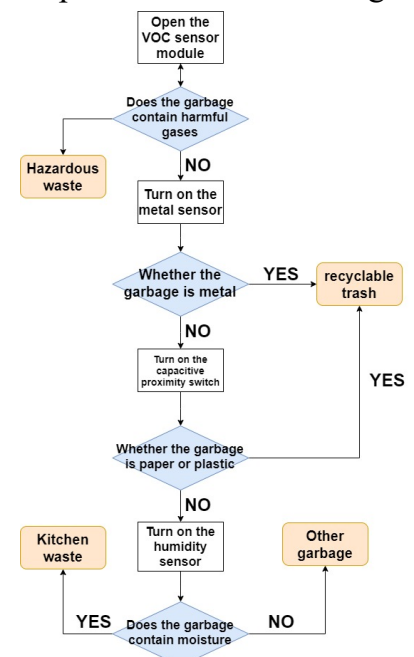

Fig.6. Auxiliary sensor recognition flowchart

In the early stage, a large amount of data training is carried out on the machine recognition system, so that it can independently realize the correctness of a certain amount of garbage and recommend the database based on this, and then use the visual camera to capture and recognize the image of the discharged garbage. Compare and analyze with the data in the database to determine the type of garbage, and then use its own mechanical structure to sort and put it into the corresponding garbage bin $^{3}$.

If there is garbage that the garbage can cannot identify, the four auxiliary sensors are used to determine the type of garbage in real time, and the garbage is placed in the buffer area of the garbage can for manual processing to avoid misplacement. Mark the judgment result, take a photo and upload it, send it to the staff for manual review, and finally upload the result to the database to serve the next identification and judgment. When faced with this

(C) The 2021 International Conference on Artificial Life and Robotics (ICAROB2021), January 21 to 24, 2021 
kind of garbage next time, the trash can will be able to directly identify and classify through machine vision.

As the database shares the data between all smart trash cans online, the learning speed of smart trash cans will increase exponentially. The overall flow chart is shown in Figure 7.

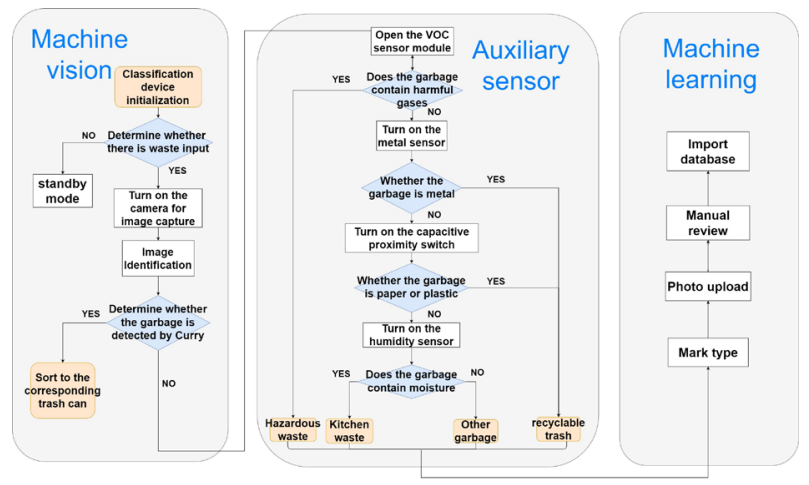

Fig.7. Overall flow chart

\section{Prospect analysis}

Through the analysis of various existing smart trash cans, the problem of insufficient machine vision recognition accuracy in the front-end collection part of the garbage classification process and cannot meet the needs of daily intelligent recognition and classification is summarized and studied. This article adopts four types of sensors. The organic combination of "new garbage" classified by auxiliary sensors is marked and then manually reviewed and entered into the database, which greatly improves the accuracy of garbage identification.

At the same time, it combines the needs of Chinese garbage classification, and expands the scope of classification to four types: recyclable, hazardous, food waste and other garbage. This classification method is based on whether it is degradable, harmful to the human body, and reusable. It has universal applicability to the garbage classification and treatment methods of various countries. Therefore, the design has been adjusted to some extent and can be applied to the global garbage classification.

In addition, placing the "new garbage" mark classified by the auxiliary sensor into the buffer area for manual processing ensures the accuracy of the classified garbage and ensures that the back-end classification and recycling can be carried out directly after the front-end collection part is completed. This saves nearly $80 \%$ of human resources, and greatly improves the efficiency of waste sorting and treatment, reducing land resources wasted due to long-term garbage stacking, and alleviating the problem of global waste sorting and treatment. Therefore, this design has excellent application prospects.

\section{Conclusion}

Aiming at the problem of insufficient accuracy of smart garbage classification, this paper proposes the use of four types of sensors to assist machine vision and machine learning recognition to improve the recognition accuracy and the correct rate of intelligent classification. Realize the classification and release of four kinds of garbage with high accuracy, greatly improve the accuracy of garbage identification, reach the standard of actual use, save a lot of waste of manpower and natural resources, and the design has the advantages of low cost and easy iteration. , Provides a new reference for the front-end collection method of garbage classification.

\section{References}

1. Golnabi, H., and A. Asadpour. Design and application of industrial machine vision systems. Robotics and Computer-Integrated Manufacturing, 2007, 23.6: 630-637.

2. Liu, Jianghai, Yadong Jiang. Design of intelligent trash can be based on machine vision. 2020 International Conference on Image, Video Processing and Artificial Intelligence. Vol. 11584. International Society for Optics and Photonics, 2020.

3. Vernon, David. Machine vision-Automated visual inspection and robot vision. STIA 92 (1991): 40499. 